\title{
A New Approach to Simulate the Wind Power Energy Based on the Probability Density Function.
}

\begin{abstract}
Electricity generate from the wind has been increased around the world. This development is related to more advantages of wind rather than conventional power plants. These advantages include the environmental and economic benefits. Beside these advantages there is some risk for investors. The intermittent and fluctuation nature of wind cannot allow the investors and operators determined the amount of these valuable energy. The term capacity value has been used for long term planning. Capacity value directly affects the generation cost. If capacity credit is low (low wind regime), the possible energy generation cost will be high and viceversa. In this paper, proposed a method to determine the capacity value by using the probability distribution function of wind speed, and then evaluate the amount of electricity generate seasonally by wind turbines in a specific area. And finally, an error correction factor will be offered for wind investors.
\end{abstract}

Keyword: Capacity value, wind speed, expected value, probability distribution function, WTGS 